\title{
¿EXISTEN DIFERENCIAS EN LAS EMOCIONES EXPERIMENTADAS POR LOS ALUMNOS DE EDUCACIÓN SECUNDARIA SEGÚN EL CURSO?
}

\author{
Ma Antonia Dávila Acedo \\ Departamento Didáctica de las Ciencias Experimentales y Matemáticas, Facultad de Educación, Universidad de \\ Extremadura. Avda. de Elvas s/n, Badajoz, España. E-mail: mdavilaacedo@unex.es \\ Ana Belén Borrachero Cortés \\ Facultad de Educación, Universidad Internacional de la Rioja, Logroño, España \\ Diego Airado Rodríguez \\ Departamento Didáctica de las Ciencias Experimentales y Matemáticas, Facultad de Educación, Universidad de \\ Extremadura. Avda. de Elvas s/n, Badajoz, España. E-mail: mdavilaacedo@unex.es
}

https://doi.org/10.17060/ijodaep.2017.n1.v2.921

Fecha de Recepción: 3 Febrero 2017

Fecha de Admisión: 1 Abril 2017

\section{RESUMEN:}

La presenta investigación surge por la necesidad de conocer y detectar las emociones que experimentan los alumnos de Educación Secundaria hacia el aprendizaje de Física y Química, pues se está produciendo una disminución en el número de alumnos en los distintos itinerarios relacionados con las Ciencias. En esta investigación se analiza y compara la evolución de las emociones que experimentan los alumnos de Educación Secundaria Obligatoria hacia el aprendizaje de Física y Química en función del curso, y si existe una relación entre las emociones y el rendimiento académico. La muestra estaba constituida por 431 alumnos de Educación Secundaria Obligatoria (ESO), distribuidos en tres cursos: $2^{\circ}, 3^{\circ}$ y $4^{\circ}$ de ESO; de distintos centros de Badajoz durante el curso escolar 2014/2015. Para realizar esta investigación, se elaboró un cuestionario donde el alumno señalaba con qué frecuencia experimentaba emociones tanto positivas como negativas hacia el aprendizaje de Física y Química. Los resultados muestran que existe un descenso en la frecuencia media de emociones positivas al pasar del $2^{\circ}$ al $4^{0}$ curso de ESO. En cambio, se produce un aumento en la frecuencia media de las emociones negativas al pasar de $2^{\circ}$ a $4^{\circ}$ de ESO.

Palabras Claves: Emociones, Física y Química, Alumnos, Educación Secundaria, Curso, Rendimiento académico.

\section{ABSTRACT:}

The present research arises for the need to know and detect the emotions that Compulsory Secondary Education students experienced towards the learning of Physics and Chemistry, because there is a decrease in the number of students at the different itineraries related to the science. This 
research analyzed and compared the evolution of emotions experienced by the ESO students to learning Physics and Chemistry according to the grade, and if there is a relationship between the emotions and the academic performance. The sample consisted of 431 students of Compulsory Secondary Education (ESO) from different schools in Badajoz during the 2014/2015 academic year. A descriptive methodology by survey was used for performing this research. The participants completed a questionnaire anonymously about emotions experienced during the learning in the field of Physics and Chemistry and the frequency, as well as the marks they get. The results showed that the students of Compulsory Secondary Education (ESO) experienced a decrease in the average frequency of positive emotions from $2^{\text {nd }}$ to $4^{\text {th }}$ ESO level. On the other hand, negative emotions increased from $2^{\text {nd }}$ to $4^{\text {th }}$ ESO level. In addition, an increases in average frequency of positive emotions as the academic performance increases.

Key Words: Emotions, Contents, Physics and Chemistry; Learning; Secondary.

\section{ANTECEDENTES}

El concepto de emoción ha sido estudiado por diferentes líneas de investigación desde distintas perspectivas, pero todas manifiestan que se trata de un proceso complejo que analiza las reacciones subjetiva ante una situación o evento personal, que conlleva cambios fisiológicos y en la conducta (Bisquerra, 2003; Kelchtermans y Deketelaere, 2016; Mellado et al., 2014) y condicionando de este modo nuestro estado de ánimo (Casacuberta, 2000), como forma básica de regulación, adaptación y supervivencia (Darwin, 1872).

Existen numerosas taxonomías para clasificar las emociones, pero si lo centramos al efecto de las emociones en el comportamiento (Bisquerra, 2000; Goleman, 1996), pueden clasificarse en dos tipos de emociones: positivas y negativas. Las emociones positivas producen sentimientos agradables, con duración temporal corta. En cambio, las negativas implican un sentimiento desagradable (Fernández-Abascal, Martín y Domínguez, 2001).

Hoy en día, el estudio de las emociones ha adquirido gran importancia y gracias a multitud de estudios (Gardner, 2005; Hargreaves, 2003; Punset, 2010; Sutton y Wheatley, 2003), puede afirmarse que tanto los aspectos cognitivos como los afectivos influyen en el proceso de enseñanzaaprendizaje, pues el mundo subjetivo y emocional que cada persona desarrolla sobre la realidad exterior da sentido a las relaciones y hace comprender el lugar propio que se ocupa en el mundo. Trabajos recientes se han centrado en la importancia de las emociones en la enseñanza de las ciencias en particular (Hong, Lin y Lawrenz, 2012; Hugo, 2008; Mellado et al., 2014)

Por tanto, es necesario conocer los aspectos emocionales que experimentan los alumnos de secundaria, a través de distintas actividades y estrategias de enseñanza, pues las emociones positivas favorecen el aprendizaje, mientras que las emociones negativas limitan la capacidad de aprender.

Asimismo, conociendo las actitudes de los estudiantes hacia las ciencias, en concreto hacia la Física y Química, los profesores pueden reflexionar y planificar el proceso de enseñanza/aprendizaje de forma que resulte más eficaz (Cheung, 2011).

Por todo ello, puede decirse que las emociones condicionan el aprendizaje hacia las ciencias. En Educación Secundaria, estas emociones dependen del contenido a tratar, siendo más positivas hacia las ciencias naturales y más negativas hacia Física y Química (Brígido, Couso, Gutiérrez y Mellado, 2013). Además, se desprende la idea de que las emociones hacia las ciencias van cambiando a la vez que aumenta la edad de los alumnos, pues las emociones negativas son más notables en la etapa de Educación Secundaria que en los ciclos de Educación Primaria (Beauchamp y Parkinson, 2008). 
Además, la etapa de Educación Secundaria Obligatoria es una etapa importante porque en ella se deciden los itinerarios posteriores e incluso las futuras carreras universitarias. En línea de estos antecedentes, en esta investigación se pretende conocer las emociones tanto positivas como negativas que experimentan los alumnos de Educación Secundaria Obligatoria en el aprendizaje de Física y Química en función del curso.

De este modo, conociendo lo que ocurre día a día en las aulas de Secundaria se puede ayudar a eliminar esa visión negativa que tienen hacia esta materia e intervenir en un futuro para mejorar el aprendizaje hacia las ciencias.

\section{OBJETIVOS}

Con esta investigación se pretenden lograr los siguientes objetivos:

Conocer las emociones que experimentan los alumnos de $2^{\circ}, 3^{\circ}$ y $4^{0}$ de Educación Secundaria Obligatoria hacia el aprendizaje de Física y Química.

Encontrar diferencias en las emociones tanto positivas como negativas experimentadas por los alumnos de Educación Secundaria según el curso.

\section{MUESTRA}

El proceso de muestreo que se ha llevado a cabo para seleccionar a los alumnos encuestados ha sido un muestreo no probabilístico de conveniencia o incidental. Las razones de esta decisión se deben a la disponibilidad de tiempo y de casos.

La muestra está constituida por un total de 431 alumnos de Educación Secundaria Obligatoria (ESO) de distintos centros, tanto públicos como concertados, de la ciudad de Badajoz durante el

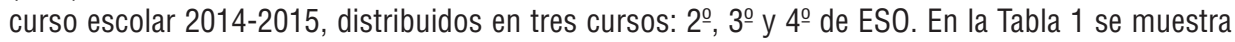
la distribución del alumnado por cursos.

Tabla 1. Distribución del alumnado por cursos

\begin{tabular}{ccc}
\hline Curso & $\mathbf{N}^{\circ}$ Alumnos & Porcentaje \\
\hline $2^{\circ}$ E.S.O & 149 & $34.6 \%$ \\
\hline $3^{\circ}$ E.S.O & 152 & $35.3 \%$ \\
\hline $4^{\circ}$ E.S.O & 130 & $30.2 \%$ \\
\hline
\end{tabular}

El $47.1 \%$ de la muestra son mujeres y $52.9 \%$ son hombres. Las edades de los alumnos oscilan entre los 13 y 17 años, situándose la media en torno a los 14-15 años.

\section{METODOLOGÍA DE INVESTIGACIÓN}

Para realizar esta investigación se ha utilizado una metodología descriptiva por encuesta, también denominada no experimental. El instrumento de recogida de datos fue un cuestionario de elaboración propia teniendo en cuenta algunas ideas del cuestionario de Borrachero (2015), en el que se recogen opiniones manifestadas por los futuros profesores de Secundaria sobre el recuerdo de las emociones hacia la Física y Química durante su período de aprendizaje.

Los participantes cumplimentaron un cuestionario totalmente anónimo sobre las emociones experimentadas en el aprendizaje de Física y Química, a lo largo del curso académico.

En la Tabla 2 se muestran las siete emociones positivas y las siete emociones negativas selec- 
cionadas para llevar a cabo esta investigación. Estas emociones han sido medidas a través de una escala de puntuación de tipo Likert de 11 puntos donde "0=Nunca" y "10=Máxima frecuencia". Estas emociones han sido seleccionadas a partir de las categorizaciones realizadas por diversos autores (Bisquerra, 2009; Borrachero, 2015; Casacuberta, 2000; Damasio, 2010; Francisco, Gervás y Hervás, 2005). Se ha obtenido un Coeficiente de Fiabilidad de Cronbach de .824 tanto para las emociones positivas como negativas.

Tabla 2.

Clasificación de las emociones.

\begin{tabular}{|c|c|c|c|}
\hline & \multicolumn{3}{|c|}{ Clasificación de las emociones } \\
\hline \multirow{7}{*}{ 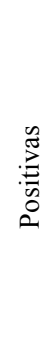 } & Alegría & \multirow{7}{*}{ 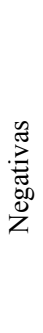 } & Aburrimiento \\
\hline & Confianza & & Ansiedad \\
\hline & Diversión & & Asco \\
\hline & Entusiasmo & & Miedo \\
\hline & Satisfacción & & Nerviosismo \\
\hline & Sorpresa & & Preocupación \\
\hline & Tranquilidad & & Tristeza \\
\hline
\end{tabular}

Una vez seleccionado el centro participante en esta investigación, se solicitó la colaboración de los profesores responsables de los cursos para pasar los cuestionarios en los días y horas posibles. Los alumnos tardaron, aproximadamente, unos 30 minutos en rellenar el cuestionario. Se mostraron curiosos e interesados por el contenido del mismo.

Una vez recogidos los datos de los cuestionarios completados por los alumnos, fueron procesados y analizados estadísticamente en sistema informático mediante el paquete estadístico SPSS 19 (Statistical Package for the Social Science). En el análisis estadístico de los datos se trabaja con un nivel de confianza del 95\%.

Se ha realizado la Prueba ANOVA de un factor para comparar la varianza y pruebas Post-Hoc para determinar entre qué cursos se dan más diferencias significativas en las emociones experimentadas por los alumnos de Educación Secundaria Obligatoria hacia el aprendizaje de Física y Química, comparando las puntuaciones medias de las variables cuantitativas de los tres grupos establecidos.

\section{RESULTADOS Y DISCUSIÓN}

En este apartado, se muestran los resultados obtenidos tras realizar el análisis tanto descriptivo como el análisis inferencial de las emociones que experimentan los alumnos de ESO en el aprendizaje de Física y Química, en función del curso; así como la relación con el rendimiento académico.

\section{Análisis Descriptivo \\ Emociones experimentadas por los alumnos de Educación Secundaria hacia el aprendizaje de Física y Química.}

En las Tablas 3, 4 y 5 se muestran los estadísticos descriptivos de las emociones tanto positivas como negativas, experimentadas por los alumnos hacia el aprendizaje de Física y Química, 
según el curso. Donde la frecuencia de estas emociones han sido medidas a través de una escala tipo Likert de puntuación, donde 0 es "Mínima Frecuencia" y 10 "Máxima Frecuencia".

Puede observarse para el segundo curso de ESO que la frecuencia media más alta en emociones positivas se encuentra en alegría $(6,78)$, seguida de satisfacción $(6,42)$ y confianza $(6,41)$. Sin embargo, en emociones negativas la frecuencia media más alta está en preocupación $(5,24)$, seguida de nerviosismo $(4,88)$ y aburrimiento $(4,63)$.

Tabla 3.

Estadísticos descriptivos de las emociones positivas y negativas experimentadas por los alumnos de $2^{\circ}$ de ESO hacia el aprendizaje de Física y Química.

\begin{tabular}{|c|c|c|c|c|c|c|c|c|c|c|c|}
\hline & Emociones & $\mathbf{n}$ & Mín. & Máx. & $\overline{\mathbf{x}}$ & & Emociones & $\mathbf{n}$ & Mín. & Máx. & $\overline{\mathbf{x}}$ \\
\hline \multirow{7}{*}{$\sum_{0}^{\infty}$} & Alegría & 149 & 0 & 10 & 6,78 & \multirow{7}{*}{$\sum_{\substack{\mid c \\
z}}^{\sum_{1}^{\infty}}$} & Aburrimiento & 149 & 0 & 10 & 4,63 \\
\hline & Confianza & 149 & 0 & 10 & 6,41 & & Ansiedad & 149 & 0 & 10 & 2,91 \\
\hline & Diversión & 149 & 0 & 10 & 6,17 & & Asco & 149 & 0 & 10 & 2,55 \\
\hline & Entusiasmo & 149 & 0 & 10 & 5,93 & & Miedo & 149 & 0 & 10 & 2,65 \\
\hline & Satisfacción & 149 & 0 & 10 & 6,42 & & Nerviosismo & 149 & 0 & 10 & 4,88 \\
\hline & Sorpresa & 149 & 0 & 10 & 4,91 & & Preocupación & 149 & 0 & 10 & 5,24 \\
\hline & Tranquilidad & 149 & 0 & 10 & 6,26 & & Tristeza & 149 & 0 & 10 & 2,49 \\
\hline
\end{tabular}

En el caso del tercer curso de ESO la frecuencia media más alta en emociones positivas se encuentra en satisfacción $(6,36)$, seguida de alegría $(6,29)$ y confianza $(6,14)$. Sin embargo, en emociones negativas la frecuencia media más alta está en preocupación $(6,35)$, seguida del nerviosismo $(5,69)$ y el aburrimiento $(5,08)$.

Tabla 4.

Estadísticos descriptivos de las emociones positivas y negativas experimentadas por los alumnos de $3^{\circ}$ de ESO hacia el aprendizaje de Física y Química.

\begin{tabular}{|c|c|c|c|c|c|c|c|c|c|c|c|}
\hline & Emociones & $\mathbf{n}$ & Mín. & Máx. & $\overline{\mathbf{x}}$ & & Emociones & n & Mín. & Máx. & $\overline{\mathbf{x}}$ \\
\hline \multirow{7}{*}{$\sum_{0}^{\infty}$} & Alegría & 152 & 0 & 10 & 6,29 & \multirow{7}{*}{$\sum_{\substack{|c| \\
Z}}^{\sum_{Z}^{\infty}}$} & Aburrimiento & 152 & 0 & 10 & 5,08 \\
\hline & Confianza & 152 & 0 & 10 & 6,14 & & Ansiedad & 152 & 0 & 10 & 3,56 \\
\hline & Diversión & 152 & 0 & 10 & 5,18 & & Asco & 152 & 0 & 10 & 2,64 \\
\hline & Entusiasmo & 152 & 0 & 10 & 5,46 & & Miedo & 152 & 0 & 10 & 3,46 \\
\hline & Satisfacción & 152 & 0 & 10 & 6,36 & & Nerviosismo & 152 & 0 & 10 & 5,69 \\
\hline & Sorpresa & 152 & 0 & 10 & 5,14 & & Preocupación & 152 & 0 & 10 & 6,35 \\
\hline & Tranquilidad & 152 & 0 & 10 & 5,09 & & Tristeza & 152 & 0 & 10 & 2,88 \\
\hline
\end{tabular}

Por otro lado, en el cuarto curso de ESO la frecuencia media más alta en emociones positivas se encuentra en satisfacción $(6,26)$, confianza $(5,63)$ y alegría $(5,33)$. Sin embargo, en emociones negativas la frecuencia media más alta está en preocupación $(6,70)$, seguida del aburrimiento $(6,25)$. 
Tabla 5.

Estadísticos descriptivos de las emociones positivas y negativas experimentadas por los alumnos de $4^{0}$ de ESO hacia el aprendizaje de Física y Química.

\begin{tabular}{|c|c|c|c|c|c|c|c|c|c|c|c|}
\hline & Emociones & $\mathbf{n}$ & Mín. & Máx. & $\overline{\mathbf{x}}$ & & Emociones & $\mathbf{n}$ & Mín. & Máx. & $\overline{\mathbf{x}}$ \\
\hline \multirow{7}{*}{$\frac{\sum^{\infty}}{3}$} & Alegría & 130 & 0 & 10 & 5,33 & \multirow{7}{*}{$\sum_{\substack{\mid c \\
z}}^{\infty}$} & Aburrimiento & 130 & 0 & 10 & 6,25 \\
\hline & Confianza & 130 & 0 & 10 & 5,63 & & Ansiedad & 130 & 0 & 10 & 4,18 \\
\hline & Diversión & 130 & 0 & 10 & 4,49 & & Asco & 130 & 0 & 10 & 3,89 \\
\hline & Entusiasmo & 130 & 0 & 10 & 5,57 & & Miedo & 130 & 0 & 10 & 4,70 \\
\hline & Satisfacción & 130 & 0 & 10 & 6,26 & & Nerviosismo & 130 & 0 & 10 & 6,02 \\
\hline & Sorpresa & 130 & 0 & 10 & 5,10 & & Preocupación & 130 & 0 & 10 & 6,70 \\
\hline & Tranquilidad & 130 & 0 & 10 & 4,37 & & Tristeza & 130 & 0 & 10 & 3,76 \\
\hline
\end{tabular}

A partir de la puntuación media de los tres curso objeto de estudio, puede decirse que existen diferencias en las emociones experimentadas por los alumnos de $2^{\circ}, 3^{\circ}$ y $4^{\circ}$ de ESO. Se produce un descenso en la frecuencia media de emociones positivas al pasar de $2^{0}$ a $4^{\circ}$ de ESO.

En cambio, en las emociones negativas se produce un incremento en la frecuencia media al pasar de $2^{0}$ a $4^{\circ}$ de ESO. También encontramos significativo que las emociones negativas preocupación y aburrimiento se encuentran presentes con mayor frecuencia en los tres cursos estudiados.

\section{Análisis Inferencial}

Emociones positivas experimentadas por los alumnos de Educación Secundaria según el curso.

En la Figura 1 se representa la puntuación media de la frecuencia de las emociones positivas experimentadas por los alumnos de $2^{0}, 3^{0}$ y $4^{0}$ de ESO hacia el aprendizaje de Física y Química, según el curso. Para medir las emociones se ha utilizado la media de cada una de las emociones dentro de un escala de 0 (Mínima frecuencia) a 10 (Máxima frecuencia).

Figura 1.

Frecuencia media de las emociones positivas experimentadas por los alumnos de Secundaria ante el aprendizaje de Física y Química según el curso.

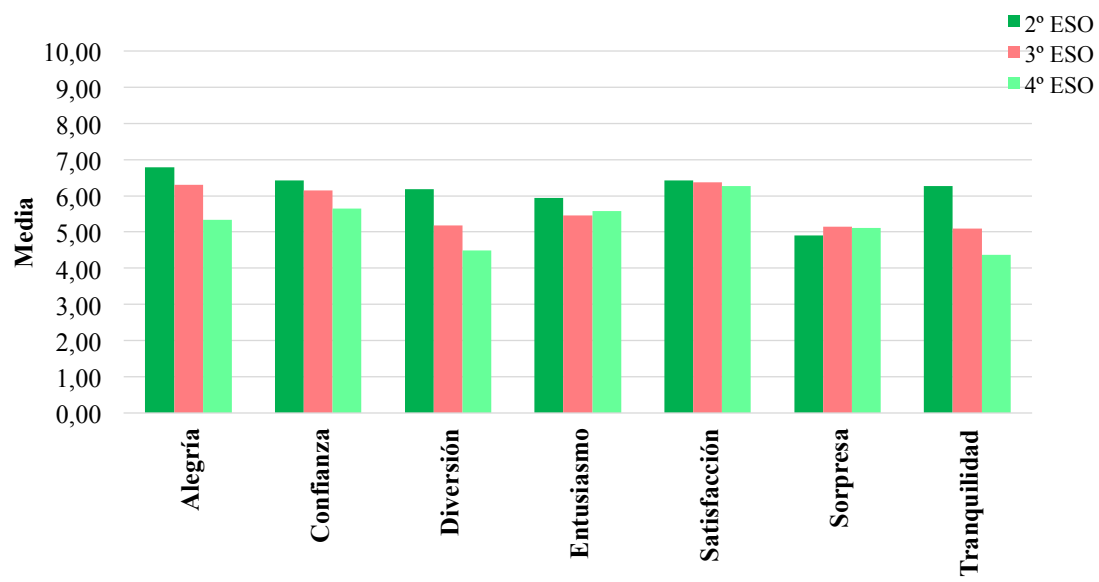


Como puede observarse la frecuencia media de las emociones positivas va disminuyendo a medida que se avanza en el curso escolar.

Seguidamente se ha realizado la Prueba ANOVA de un factor para comprobar si existen diferencias significativas en la frecuencia de las emociones positivas experimentadas por los alumnos y el curso, tras el aprendizaje de Física y Química

En la Tabla 6 se muestran los resultados obtenidos tras realizar la Prueba, puede observarse que existe diferencias significativas en el aprendizaje de Física y Química, y el curso donde se encuentren, en la frecuencia media de las emociones positivas Alegría $(p \geq .000)$, Confianza $(p=.045)$, Diversión $(p \geq .000)$ y Tranquilidad $(p \geq .000)$.

Tabla 6.

Prueba ANOVA de un factor entre el curso y emociones positivas experimentadas en el aprendizaje de Física y Química.

\begin{tabular}{ccc}
\hline \hline Emociones & F & Sig. \\
\hline Alegría & 9.392 & $.000^{* *}$ \\
Confianza & 2.756 & $.045^{*}$ \\
Diversión & 9.146 & $.000^{* *}$ \\
Entusiasmo & .940 & .392 \\
Satisfacción & .128 & .880 \\
Sorpresa & .208 & .812 \\
Tranquilidad & 12.164 & $.000^{* *}$ \\
\hline
\end{tabular}

${ }^{*} \mathrm{p} \leq .010 . \quad{ }^{*} \mathrm{p} \leq .050 . \mathrm{F}=$ estadístico de contraste. Sig. = significación estadística

Seguidamente, se ha realizado las pruebas Post-Hoc de Tukey (HDS de Tukey) con el fin de establecer entre qué grupos existen más diferencia significativas, en este caso en qué cursos se producen mayores diferencias. En el caso de $2^{0}$ y $3^{0}$ de Educación Secundaria Obligatoria no hay diferencias en la frecuencia media de emociones positivas, mientras que en el cuarto curso de secundaria la frecuencia media de estas emociones disminuye, encontrándose más diferencias en emociones tales como alegría, confianza, diversión y tranquilidad.

Emociones negativas experimentadas por los alumnos de Educación Secundaria según el curso.

En la Figura 2 se representa la puntuación media de la frecuencia de las emociones positivas experimentadas por los alumnos de $2^{0}, 3^{\circ}$ y $4^{0}$ de ESO hacia el aprendizaje de Física y Química, según el curso. Para medir las emociones se ha utilizado la media de cada una de las emociones dentro de un escala de 0 (Mínima frecuencia) a 10 (Máxima frecuencia). 
Figura 2.

Frecuencia media de las emociones negativas experimentadas por los alumnos de Secundaria ante el aprendizaje de Física y Química según el curso.

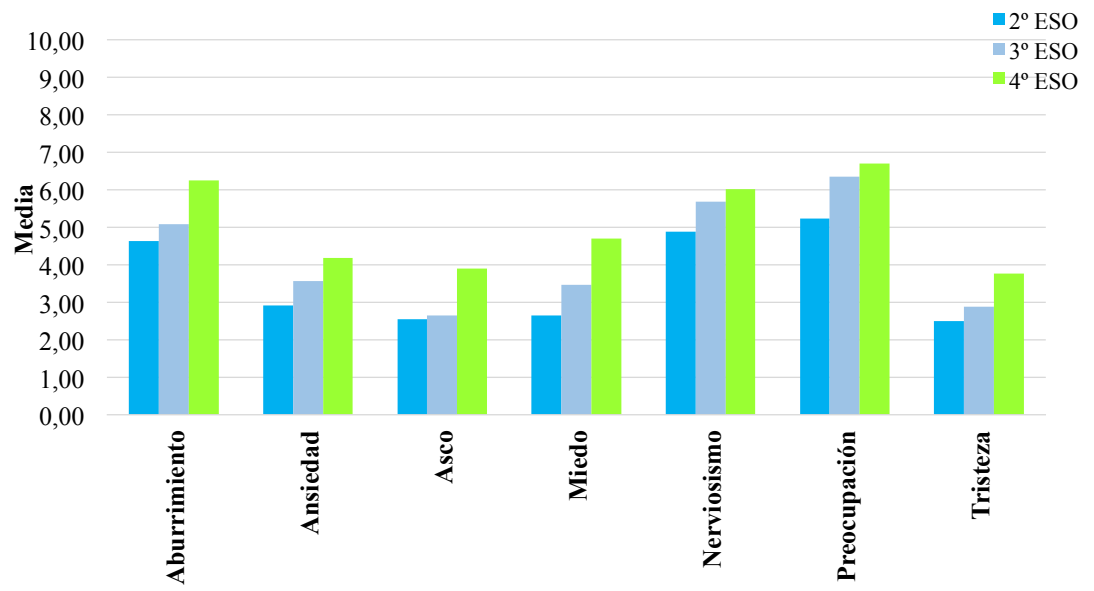

Como puede observarse la frecuencia media de las emociones negativas va aumentando a medida que se avanza en el curso escolar.

Seguidamente se ha realizado la Prueba ANOVA de un factor para comprobar si existen diferencias significativas en la frecuencia de las emociones negativas experimentadas por los alumnos y el curso, tras el aprendizaje de Física y Química.

En la Tabla 7 se muestran los resultados obtenidos tras realizar la Prueba, puede observarse que existe diferencias significativas en aprendizaje de Física y Química y el curso donde se encuentren, en la frecuencia media de las emociones negativas Aburrimiento $(p \geq .000)$, Ansiedad $(p=.010)$, Asco ( $p=.002)$, Miedo $(p \geq .000)$, Nerviosismo $(p=.014)$, Preocupación $(p \geq .000)$ y Tristeza $(p=$ $.003)$.

Tabla 7.

Prueba ANOVA de un factor entre el curso y emociones negativas experimentadas en el aprendizaje de Física y Química.

\begin{tabular}{ccc}
\hline \hline Emociones & F & Sig. \\
\hline Aburrimiento & 8.943 & $.000^{* *}$ \\
Ansiedad & 4.663 & $.010^{* *}$ \\
Asco & 6.090 & $.002^{* *}$ \\
Miedo & 12.179 & $.000^{* *}$ \\
Nerviosismo & 4.309 & $.014^{*}$ \\
Preocupación & 9.080 & $.000^{* *}$ \\
Tristeza & 6.073 & $.003^{* *}$ \\
\hline
\end{tabular}


Además, se ha realizado las pruebas Post-Hoc de Tukey (HDS de Tukey) con el fin de establecer entre qué grupos existen más diferencia significativas, en este caso en qué cursos se producen mayores diferencias. Como puede observarse la frecuencia media de emociones negativas, experimentadas por los alumnos, como aburrimiento, asco, miedo y tristeza aumentan de forma significativa de $2^{\circ}$ a $3^{\circ}$ y de $3^{\circ}$ a $4^{\circ}$ de Educación Secundaria Obligatoria, mientras que la frecuencia media de las emociones ansiedad, nerviosismo y preocupación aumenta de manera significativa de $2^{\circ}$ a $4^{\circ}$ de ESO.

A partir de los resultados obtenidos, puede decirse que resulta necesario conocer las emociones que experimentan los alumnos de ESO hacia la materia de Física y Química. En nuestro estudio las emociones negativas tales como nerviosismo y preocupación son claves en el tercer y cuarto curso de ESO, pues para muchos alumnos la preocupación les puede condicionar a la elección o no de esta materia en los próximos cursos, y el nerviosismo les provocará una gran inseguridad a la hora de enfrentarse a la materia con una actitud más positiva.

Esto resultados coinciden con la investigación realizada por Dávila, Borrachero, Cañada y Sánchez (2016) con una muestra de alumnos de Educación Secundaria de dos centro de la provincia de Badajoz durante el curso académico 2013/2014, donde se produce una disminución en la frecuencia media de emociones positivas tales como alegría, confianza, felicidad, tranquilidad y entusiasmo al pasar de $2^{\circ}$ a $4^{\circ}$ de ESO. En cambio, la frecuencia media de emociones negativas como aburrimiento, preocupación y asco se incrementan al pasar de $2^{0}$ al $3^{0}$ curso de ESO.

\section{CONCLUSIONES}

La investigación realizada sobre las emociones que experimentan los alumnos de Educación Secundaria Obligatoria, muestra la necesidad de detectar estas emociones en las clases de ciencias, con el fin de mejorar esa visión tan negativa hacia estas materias.

Se ha comprobado la existencia de diferencias significativas en la frecuencia media de las emociones tanto positivas como negativas experimentadas por los alumnos ESO y el curso que realizan. De este modo, los alumnos experimentan una menor frecuencia de emociones positivas como alegría, confianza, diversión y tranquilidad, al pasar de $2^{\circ}$ a $4^{0}$ de ESO. Este aspecto es muy preocupante para el aprendizaje de las ciencias, ya que el $4^{\circ}$ curso es decisivo, pues los alumnos deciden su futuro itinerario de Bachillerato. Para autores como Murphy y Beggs (2003) todo esto depende de la formación académica impartida y recibida, ya que puede contribuir al desarrollo de actitudes negativas y desinterés hacia las ciencias.

Por otro lado, se observa que los alumnos experimentan con mayor frecuencia emociones negativas como aburrimiento, asco, miedo y tristeza siendo incremento mayor al pasar de $2^{\circ}$ a $3^{\circ}$, mientras que el incremento en la frecuencia media de la emoción negativa ansiedad en mayor de $2^{\circ}$ a $4^{\circ}$ de ESO. Esto puede ser debido a que en $2^{-}$de ESO se imparten contenidos mayoritariamente relacionados con la Biología y Geología, mientras que el 3ํㅡㄹ ESO son de Física y Química. En este sentido, el estudio realizado por Brígido, Borrachero, Bermejo y Mellado (2013) con futuros maestros de Educación Primaria, concluye que las emociones positivas hacia las Ciencias de la Naturaleza disminuyen de Educación Primaria a Secundaria, cobrando mayor importancia las emociones negativas.

Además, otras investigaciones demuestran que se produce un declive de las actitudes positivas hacia las ciencias de Primaria a Secundaria, es decir, a medida que se avanza en el curso escolar las actitudes de los alumnos hacia las ciencias disminuyen (Osborne, Simón y Collins, 2003; Vázquez y Manassero, 2011).

Por tanto, el estudio del dominio afectivo en la asignatura de Física y Química resulta necesario, 
pues favorecer el desarrollo de actitudes positivas, a través del fomento de sentimientos y emociones positivas, mejorará las expectativas hacia esta materia, aunque en ocasiones esto resulte algo difícil, pues las habilidades afectivas y los comportamientos no son fáciles de identificar, cuantificar y evaluar (Brígido, Borrachero, Bermejo y Dávila, 2014).

Así, estudios realizados con futuros maestros de Educación Primaria indican un aumento en las emociones positivas y una disminución en las negativas experimentadas, ante la realización de actividades prácticas en el aula, produciéndose una mejora en las competencias científicas y mayor motivación por el aprendizaje en la materia. (Dávila, Borrachero, Cañada, Martínez y Sánchez, 2015).

Por todo ello, es necesario que los profesores sean capaces de detectar estas emociones en las clases en su práctica diaria y utilizar diferentes estrategias de enseñanza para que los alumnos participen de forma activa e interactúen en su aprendizaje.

\section{AGRADECIMIENTOS:}

Este trabajo ha sido financiado por el proyecto de investigación EDU2016-77007-R y beca predoctoral del Ministerio de Economía y Competitividad de España. Se agradece la ayuda al grupo GR15009 del Gobierno de Extremadura (España) y al Fondo Europeo de desarrollo regional.

\section{BIBLIOGRAFÍA}

Beauchamp, G. y Parkinson, J. (2008). Pupils' attitudes towards school science as they transfer from an ICT-rich primary school to a secondary school with fewer ICT resources: Does ICT matter? Education and Information Technologies, 13 (2), 103-118.

Bisquerra, R. (2000). Educación emocional y bienestar. Barcelona: Praxis.

Bisquerra, R. (2003). Educación emocional y competencias básicas para la vida. Revista de Investigación Educativa, 21 (1), 7-43.

Bisquerra, R. (2009). Psicopedagogía de las emociones. Madrid: Síntesis

Borrachero, A.B. (2015). Las emociones en la enseñanza y el aprendizaje de las ciencias en educación secundaria. Tesis doctoral. Facultad de Educación. Badajoz: Universidad de Extremadura.

Brígido, M., Borrachero, A. B., Bermejo, M. L. y Mellado, V. (2013). Prospective primary teachers' self-efficacy and emotions in science teaching. European Journal of Teacher Education, 36 (2), 200-217.

Brígido, M., Borrachero, A. B., Bermejo, M. L. y Dávila, M. A. (2014). Programa de intervención para la mejora de las creencias de autoeficacia en las clases de ciencias. International Journal of Developmental and Educational Psychology. INFAD Revista de Psicología, 5 (1), 73-80.

Brígido, M., Couso, D., Gutiérrez, C. y Mellado, V. (2013). The Emotions about Teaching and Learning Science: A Study of Prospective Primary Teachers in Three Spanish Universities. Journal of Baltic Science Education, 12(3), 299-311.

Casacuberta, D. (2000). Qué es una emoción. Barcelona: Crítica.

Cheung, D. (2011). Evaluating Student Attitude toward Chemistry Lessons to Echance Teaching in Secondary School. Educación Química. 22 (2), 117-122.

Damasio, A. (2010). Y el cerebro creó al hombre. Barcelona: Editorial Destino.

Darwin, C. (1872). The expression of the emotions in man and animals. London: John Murray.

Dávila, M.A., Borrachero, A.B., Cañada, F., y Sánchez, J. (2016). Evolution of emotion in learning Physics and Chemistry in Secondary Education. En Edmund Harvey (Ed.), Secondary Education. Perspective, Global Issues and Challenges (pp. 115-132) Nueva York: Nova Publishers.

Dávila, M.A., Cañada, F., Sánchez, J. y Mellado, V. (2015). Las emociones en el aprendizaje de Física y Química en Educación Secundaria. Causas relacionadas con el estudiante. Educación Química, 27(3), 217-225. 
Fernández-Abascal, E., Martín, M. y Domínguez, J. (2001). Procesos psicológicos. Madrid: Ediciones Pirámide.

Francisco, V., Gervás, P. y Hervás, R. (2005). Análisis y síntesis de expresión emocional en cuentos leídos en voz alta. Procesamiento del Lenguaje Natural, 35, 293-300.

Gardner, H. (2005). Las cinco mentes del futuro: Un ensayo educativo. Barcelona: Paidos.

Goleman, D. (1996). Inteligencia emocional. Barcelona: Cairos.

Hargreaves, A. (2003). Teaching in the knowledge society. Maidenhead: Open University Press.

Hong, Z.R., Lin, H.S. y Lawrenz, F.P. (2012). Effects of an Integrated Science and Societal Implication Intervention on Promoting Adolescents' Positive Thinking and Emotional Perceptions in Learning Science. International Journal of Science Education, 34(3), 329-252.

Hugo, D.V. (2008). Análisis del proceso de autorregulación de las Prácticas Docentes de futuras profesoras de ciencia focalizado en sus emociones. Tesis Doctoral inédita. Universidad Autónoma de Barcelona.

Kelchtermans, G.,y Deketelaere, A. (2016). The Emotional Dimension in Becoming a Teacher. In International Handbook of Teacher Education (pp. 429-461). Springer Singapore.

Mellado, V., Borrachero, A. B., Brígido, M., Melo, L. V., Dávila, M. A., Cañada, F., Conde, M. C., Costillo, E., Cubero, J., Esteban, R., Martínez, G., Ruiz, C., Sánchez, J., Garritz, A., Mellado, L., Vázquez, B., Jiménez, R. y Bermejo, M. L. (2014). Las emociones en la enseñanza de las ciencias. Enseñanza de las Ciencias, 32 (3), 11-36.

Murphy, C. y Beggs. J. (2003). Children perceptions of school science. School Science Review, 84 (308), 109-116.

Osborne, J., Simon, S. y Collins, S. (2003). Attitudes towards science: a review of the literature and its implications. International Journal of Science Education. 25 (9), 1049-1079.

Punset, E. (2010). Viaje a las emociones. Barcelona: Destino.

SPSS (2012). SPSS 22. Developer's guide. IBM, Armonk, New York.

Sutton, R. y Wheatley, K. (2003). Teachers emotions and teaching: A review of the literature and directions for future research. Educational Psychology Review, 15, 327-358.

Vázquez, A. y Manassero, M. A. (2011). El descenso de las actitudes hacia la ciencia de chicos y chicas en la educación obligatoria. Ciencia \& Educaçao, 17 (2), 249-269. 
